\title{
Non-traditional lifestyles and prevalence of mental disorders in adolescents in Goa, India
}

\author{
Aravind Pillai, Vikram Patel, Percy Cardozo, Robert Goodman, Helen A. Weiss and Gracy Andrew
}

\section{Background}

Adolescents comprise a fifth of the population of India, but there is little research on their mental health. We conducted an epidemiological study in the state of Goa to describe the current prevalence of mental disorders and its correlates among adolescents aged between 12 and 16 years.

\section{Aims}

To estimate the prevalence and correlates of mental disorders in adolescents.

\section{Method}

Population-based survey of all eligible adolescents from six urban wards and four rural communities which were randomly selected. We used a Konkani translation of the Development and Well-Being Assessment to diagnose current DSM-IV emotional and behavioural disorders. All adolescents were also interviewed on socio-economic factors, education, neighbourhood, parental relations, peer and sexual relationships, violence and substance use.

\section{Results}

Out of 2684 eligible adolescents, 2048 completed the study. The current prevalence of any DSM-IV diagnosis was $1.81 \%$; $95 \% \mathrm{Cl} 1.27-2.48$. The most common diagnoses were anxiety disorders $(1.0 \%)$, depressive disorder $(0.5 \%)$, behavioural disorder (0.4\%) and attention-deficit hyperactivity disorder $(0.2 \%)$. Adolescents from urban areas and girls who faced gender discrimination had higher prevalence. The final multivariate model found an independent association of mental disorders with an outgoing 'non-traditional' lifestyle (frequent partying, going to the cinema, shopping for fun and having a boyfriend or girlfriend), difficulties with studies, lack of safety in the neighbourhood, a history of physical or verbal abuse and tobacco use. Having one's family as the primary source of social support was associated with lower prevalence of mental disorders.

\section{Conclusions}

The current prevalence of mental disorders in adolescents in our study was very low compared with studies in other countries. Strong family support was a critical factor associated with low prevalence of mental disorders, while factors indicative of adoption of a non-traditional lifestyle were associated with an increased prevalence.

\section{Declaration of interest}

None. Funding detailed in Acknowledgements.
Studies in high-income countries have shown that psychosocial health problems during adolescence, in particular depression, anxiety and substance misuse, are relatively common. ${ }^{1-6}$ Worldwide, the leading causes of disability-adjusted life years (DALYs) in 10- to 19-year-olds were unipolar major depression, accidents and falls. ${ }^{7,8}$ Adolescents face rapidly changing challenges in their social and physical environments. Their cultural beliefs, family structure and support, peer relationships and educational opportunities influence their behaviour and adjustment. Globalisation is leading to rapid changes in these sociocultural systems in lowand middle-income countries, and changing values and expectations of adolescents may influence the risk of mental disorders. ${ }^{9,10}$

Young people (aged 10-19 years) comprise more than a fifth of India's population - an estimated 230 million people. ${ }^{11}$ Although adolescent health has gained increasing prominence in India's national health policies, the focus has been on reproductive and sexual health concerns. Despite reports showing that suicide is a leading cause of death in young people in India, ${ }^{12}$ mental health has been a low priority in health policy for adolescents. The few published studies from India have reported prevalence of mental disorders from $2.6 \%$ to $35.6 \%{ }^{13-19}$ Although comparability between the findings of these studies is limited owing to methodological factors, ${ }^{5}$ one reason for the wide variation in rates could be the strong influence of social, cultural and environmental factors on the risk of mental disorders in adolescents.

Unlike many medical disorders, a majority of adolescent mental disorders represent the extreme end of normal distribution and are multifactorial in aetiology. ${ }^{20}$ Substantial research literature, mostly from developed countries, ${ }^{6,7,21}$ suggests a complex socioecological framework of risk factors operating in multiple contexts that are central to the lives of adolescents, namely, home, school, peer group and neighbourhood. ${ }^{21} \mathrm{~A}$ few studies from low- and middle-income countries have identified family structure and relationships, ${ }^{17,22,23}$ social class, ${ }^{24}$ urbanisation $^{23}$ and school failure $^{22}$ as some factors associated with mental disorders in adolescents. Substance misuse ${ }^{25}$ and physical and sexual abuse are consistently reported with markedly impaired emotional functioning. ${ }^{26,27}$ However, there have been no studies from India that have systematically examined the association of these multiple factors with adolescent mental health in a population setting. Understanding the role of these factors may help guide prevention and intervention initiatives.

We describe the findings of a population-based study aimed at strengthening the limited evidence base on factors associated with mental disorders in adolescents in low- and middle-income countries. The data reported here have been derived from the recruitment phase of a cohort study of vulnerability and resilience factors for adolescent health carried out in Goa, India. We aimed to estimate the prevalence of mental disorders, and to assess the association with factors reflected in the literature and our understanding of the sociocultural contexts of Goa, as shown in our conceptual model (Fig. 1).

\section{Methods}

\section{Setting}

The study was set in one urban and one rural community in the southern district of the state of Goa, India. Goa is one of the 
smaller states in India with a population of just over 1.4 million people living in an area of $3702 \mathrm{~km}^{2}{ }^{28}$ The rural study population lived in the catchment area of the Bali primary health centre. These villages consist mostly of a population from a lower socio-economic background, with farming being the main source of income. The total primary health centre area was divided into eight sub-centre catchment areas. We randomly selected four areas: Morpila, Barcem, Bali and Fatorpa with a population of 2781, 6040, 5000 and 5000 respectively. The urban community was located in the city of Margao (population of 44000), the largest city and commercial capital of the state. We randomly selected 6 out of 16 wards for our study, with populations between 2000 and 5000.

\section{Sample}

Our sample comprised all adolescents aged 12-16 years residing in the selected clusters. The sample was enumerated through two sources: the family registers maintained by the health centres; and eligible adolescents residing in the area who were identified during the door to door survey. In the rural areas, we identified 1046 eligible adolescents from the family health register and a further 290 (total rural sample 1336) from the survey. In the urban areas, the family registers maintained by the health centre had not been updated for several years and thus we carried out a door to door survey and enumerated 1348 adolescents. Recruitment took place between October 2002 and May 2003 by trained researchers through face to face interviews. From the final list of eligible adolescents $(n=2684), 358$ were absent from home on the mandatory three consecutive visits by the researcher. Eighty-five adolescents could not be traced and were said to have moved out of the area. Out of the 2241 adolescents who were met by the researcher, 2054 (91.1\%) consented to participate. Of these, $6 \mathrm{did}$ not complete the Development and Well-Being Assessment (DAWBA), our primary tool for the diagnosis of a mental disorder; thus, observations from only 2048 participants were available for the analyses presented in this paper. Furthermore, we were able to complete parent interviews for only $58.5 \%$ of the adolescents $(n=1198)$. The parental interview consisted of two parts - a set of questions on risk factors and a parent DAWBA. Whenever possible, the DAWBA from the parental interviews was used along with the adolescent interview to generate the DSM-IV diagnoses; for the remaining adolescents, diagnosis was based on adolescent interview alone. The risk factor data from parental interviews were not used in the analysis. Before starting the main recruitment phase, the research team developed networks and partnerships with key local people such as anganwadi workers (community child development workers), panchayat (village council) members, heads of the primary health centres and other stakeholders in the community. Community meetings were arranged for creating awareness about the project in each village.

\section{Measurement}

We developed a structured interview for assessment of risk factors, derived primarily from an interview we had used in an earlier study on health needs of adolescents in schools ${ }^{29}$ and interviews used in studies of women's health. ${ }^{30}$ The interview was structured in the following domains based on available literature from lowand high-income countries, ${ }^{13,14,31,32}$ and our experience with adolescent psychopathology.

\section{Socio-demographic factors}

Studies from Western countries, ${ }^{6,33,34}$ as well as low- and middleincome countries, ${ }^{14,35}$ have consistently reported associations

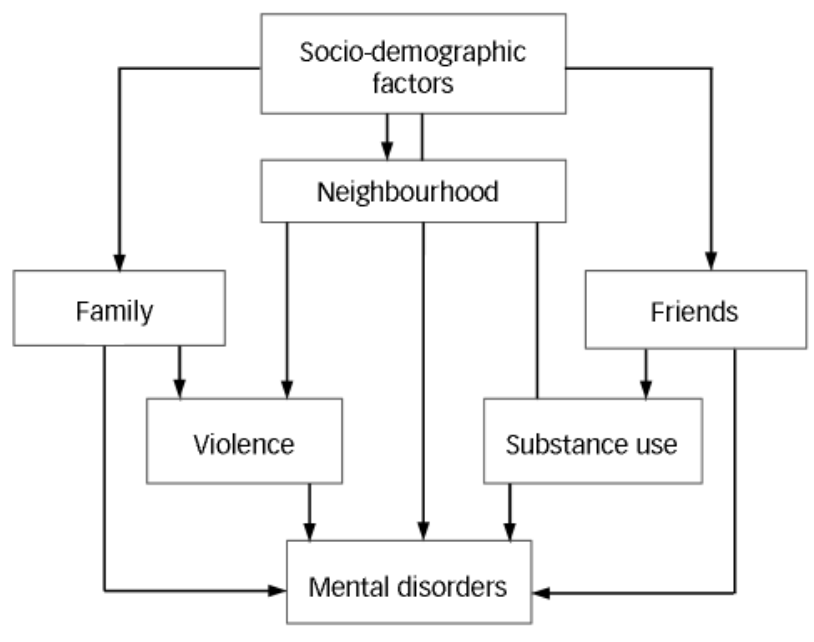

Fig. 1 Conceptual framework.

between socio-demographic factors and mental disorders among adolescents. Therefore, we included questions about gender, age, area of residence (rural/urban), language mostly spoken at home, religion, religious beliefs, who the adolescent has lived with over the past 12 months and hunger in the past 3 months due to poverty (see online table DS1). We also collected information on factors associated with schooling, namely, whether currently in school, experiencing difficulties with studies, number of days absent from school in the past 3 months and whether the adolescent had ever worked for money.

\section{Neighbourhood}

Perceptions regarding levels of safety (during the day and at night), trust (whether it was possible to borrow things and exchange favours with neighbours) and availability of health facilities (were they within an easy distance from home) (see online table DS1).

Peer relations, activities and sexual relations

Peer relationships were assessed through questions about the number of close friendships and satisfaction with regards to the number of friends. Engagement in activities was assessed by how frequently in the past 3 months the adolescent:

(a) spent time with friends;

(b) attended parties or discos;

(c) went shopping for fun (as opposed to essential shopping for the household);

(d) participated in hobbies such as listening to music;

(e) participated in community or youth club activities;

(f) participated in religious activities;

(g) went to the cinema.

Sexual relationships were extremely uncommon in this age group, thus, we asked about ever having had a boyfriend or girlfriend (i.e. a close friend of the opposite gender to whom the adolescent was sexually attracted and met regularly). Any experience of intimate physical contact such as kissing or sexual intercourse was also elicited (see online table DS2). 


\section{Family relations in past 12 months}

Parental and familial factors that were considered included:

(a) ease of communication with parents;

(b) discussing sex-related matters with parents;

(c) activities with parents;

(d) arguments with, and between, parents;

(e) perception of parents being strict, including being treated differently or being restricted from certain activities because of gender;

(f) perception of family as the first source of support when faced with four specific situations - facing a personal problem, needing to borrow a small amount of money, feeling low and needing to talk to someone, and being looked after when ill (see online table DS3).

\section{Substance use, gambling and violence}

Any use of alcohol, tobacco, gambling (playing games for money), experience of verbal and physical violence, including experience of sexual violence, in the past 12 months (see online table DS4).

\section{Psychopathology}

Psychiatric diagnoses were assessed with the DAWBA, ${ }^{36}$ an integrated package of questionnaires, interviews and rating techniques designed to generate best-estimate psychiatric diagnoses in 5- to 16-year-olds. Trained non-clinical interviewers administered a structured interview to adolescents and one parent, supplementing the structured questions with open-ended questions to get respondents to describe the problems in their own words. Experienced clinical raters (R.G., V.P.) assigned operationalised ICD-10 $10^{37}$ and DSM$\mathrm{IV}^{38}$ diagnoses after reviewing all structured and open-ended responses. Though the validity of the DSM diagnostic system has not been assessed in Goa, given its extensive use in clinical settings - including the adolescent mental health services provided by Sangath, the lead organisation in Goa for this study - this framework has been found valuable for planning service needs in the community. Primary ratings were made by one of the authors (R.G.), an experienced child and adolescent psychiatrist who developed the DAWBA and has previously supervised international surveys using the DAWBA in Bangladesh, Brazil, Britain and Russia. ${ }^{5,39-41}$ Ratings were then checked by another author (V.P.) who had specific clinical experience in Goa. Final diagnoses were made by consensus between R.G. and V.P. When individuals had a clinically significant disorder that resulted in substantial distress or social impairment but did not meet the criteria for any operationalised diagnosis, they were given a 'not otherwise specified' diagnosis such as 'anxiety disorder not otherwise specified'.

\section{Interview}

The interview was translated to and from Hindi and English by the local research team, while the Konkani translations were reviewed by bilingual experts within the project institution and by external experts in the Konkani language. Though we could not assess the validity of the Konkani language version of the DAWBA owing to resource constraints, a Bengali language version of the DAWBA was previously validated in South Asia. ${ }^{43}$ The quality of the Konkani version of DAWBA was assessed by conducting sequential interviews with 15 bilingual individuals, in random order, using both language versions one week apart and comparing scores. Interviewers were trained by Dr Bacy Fleitlich-Bilyk, who is experienced in assessing schoolchildren in Brazil using the DAWBA. ${ }^{39}$ The questionnaire and DAWBA were piloted with 56 adolescents (those attending school and those who had dropped out of school) selected from communities with similar socioeconomic status in both urban and rural areas, and revised to ensure acceptability and clarity of the questions.

\section{Ethical considerations}

The project was approved by the Independent Ethics Commission in Mumbai, the World Health Organization ethics board and the Indian Council for Medical Research. All participants joined voluntarily after giving verbal informed consent and were free to withdraw from the study at any point. Parental consent was sought before adolescent consent. In order to carry out the interviews in schools, we obtained prior permission from Goa's Director of Education and from headteachers. Adolescents having any emotional problems were offered psychological support available at the child and adolescent clinic run by Sangath. Appropriate advice was given to participants whenever other health problems were reported.

\section{Analysis}

The primary outcome - DSM-IV diagnosis of mental disorder detected by the DAWBA - was coded as a binary variable.

Eight composite variables were generated from raw variables in the interview. Principal-components analysis was used to identify the factor structure of multiple variables in a domain. Items that were significantly correlated with a specific factor were combined to generate a new composite variable. The resulting composite variables were then assessed for their face validity as constructs, based on the clinical experience of the authors and the wider networks of colleagues and collaborators in Sangath. Scores were generated for each composite variable as described below.

(a) A 'neighbourhood safety score' was computed by combining adolescent's perception of safety during the day and at night. A 'neighbourhood trust score' was computed by combining the responses to the questions regarding ability to borrow and request favours from neighbours, and the perception of trust in the neighbourhood. Higher scores indicated lower levels of safety and trust.

(b) Two scores were computed as measures of different types of activities engaged in by adolescents outside the home. A 'leisure score' was calculated from activities indicative of an outgoing, 'non-traditional' lifestyle, for example, attending parties or discos, going to the cinema and shopping for fun. A 'community activity score' was computed on the basis of engagement in community/social work, religious activities and hobbies. Higher scores indicated higher levels of involvement with these activities.

(c) The 'parental activity score' was computed by adding the scores obtained from variables assessing activities with parents, for example, going on outings, studying, socialising, watching television, participating in sports and other activities. A higher score indicated more activities involving parents.

(d) A 'gender discrimination score' was computed by combining two variables - being treated differently (e.g. compared with a sibling of the opposite gender) and being restricted from certain activities by parents because of one's gender. Participants who reported at least one of the above were considered as facing gender-based discrimination.

(e) The 'parental stress score' was computed from the variables assessing conflicts with parents and the participant's perception of their parents being strict. Higher scores indicated greater levels of parental stress.

(f) The 'family support score' was computed on the basis of variables assessing the participant's view of the family as the first 
source of support in four specific situations: facing a personal problem; needing to borrow a small amount of money; feeling low and needing to talk to someone; and being looked after when ill. Higher scores indicated higher perceived levels of family support.

Except for the parental stress, neighbourhood safety, neighbourhood trust and gender discrimination scores, which were dichotomised, other composite scores were categorised into tertiles or quartiles for analyses. Missing values were present for many variables which were not applicable to some participants (e.g. relationship with a parent of participants not living with that parent or difficulties with studies for participants not in school) these were treated as missing in analyses and the valid number of observations available for the analyses for each variable is presented in the tables.

Multiple logistic regression was used to estimate odds ratios and $95 \%$ confidence intervals. All models were adjusted for clustering of participants within households using generalised estimating equations. All analyses were performed with Stata version 8.0 for Windows. An a priori conceptual model of the determinants of adolescent mental disorder was used to guide the multivariate analysis (Fig.1). All models included a priori adjustment for age (in years), gender and area (urban and rural). We carried out stepwise logistic regression, dropping variables in each step which did not meet our criteria for significant association with the outcome $(P<0.05$ or $\mathrm{OR} \geqslant 2$ or $<0.5)$. First, association of independent variables with a mental disorder was estimated. For each domain, a multivariate model was built which included all factors significant in the first model, plus the a priori variables (age, gender and area). Variables were retained in the domain-specific multivariate analysis if their associations remained significant after adjustment for other factors in the model. The final model contained all variables significantly associated with the outcome, plus age, gender and area.

\section{Results}

The survey included approximately equal numbers of adolescents from urban $(n=992)$ and rural $(n=1056)$ areas, and of males $(n=1031)$ and females $(n=1017)$. The response rate was significantly higher in the rural areas compared with the urban areas ( $79 \%$ v. $74 \%$ respectively; $P=0.001$ ) and adolescents from urban areas were slightly older (mean age 13.49 years $v$. 13.10 years; $P=0.01)$. Siblings were interviewed for 734 participants $(35.8 \%)$. Most individuals (75\%) spoke Konkani at home and lived with both parents $(83.6 \%)$. A small proportion (3.7\%) lived with neither parent. Most adolescents $(91.6 \%)$ were full-time students at the time of the interview; $7.9 \%$ had dropped out of school and $0.5 \%$ had never had any formal education. Overall, $39.4 \%$ of adolescents reported experiencing difficulties with their studies. A total of 157 adolescents reported having worked for pay and among these $53.2 \%$ started working before the age of 14 .

\section{Prevalence of mental disorders}

A DSM-IV mental disorder was diagnosed in 37 adolescents (prevalence $1.81 \%$; 95\% CI 1.27-2.48). The most common disorders were anxiety disorders $(n=20,54.1 \%)$, depressive disorders $(n=10,27 \%)$, behavioural disorders $(n=9,24.3 \%)$ and attentiondeficit hyperactive disorder (ADHD; $n=4,10.8 \%$ ). Of the 20 individuals diagnosed with anxiety disorders, 4 had social phobia, 4 had panic disorder with agoraphobia, 4 had obsessive-compulsive disorder (of whom 1 also had a post-traumatic stress disorder), 2 had generalised anxiety disorder and 6 had an 'anxiety disorder not otherwise specified'. Of the 10 individuals with a depressive disorder, 5 had major depressive disorder and 5 had a 'depressive disorder not otherwise specified'. Of the 9 individuals diagnosed with behavioural disorders, 3 had conduct disorder, 1 had oppositional defiant disorder and 5 had 'disruptive behaviour disorder, not otherwise specified'. Of the 4 individuals with ADHD, 3 had the combined type and 1 had the predominantly inattentive type. Several adolescents had comorbid conditions: 1 with a behavioural disorder, anxiety and depression; 3 with anxiety and depression and 1 with ADHD and a behavioural disorder. We observed a higher rate of mental disorders for the sub-group of adolescents for whom we had both parent and adolescent data (2.3\%, 95\% CI $1.5-3.2$ v. $1.0 \%$, 95\% CI $0.3-1.7)$.

\section{Factors associated with mental disorder}

\section{Socio-demographic and educational factors}

There was no association of gender or age with the prevalence of a mental disorder, but those living in urban areas had significantly higher prevalence $(\mathrm{OR}=2.2,95 \% \mathrm{CI} 1.1-4.6)$. After adjusting for area, gender and age, increased prevalence of mental disorders was associated with being from English-speaking homes, nonHindu religions, working for money, difficulties with studies, and missing 3 or more days of school during the past 3 months. Multivariate analysis found that adolescents from English-speaking homes (compared with Konkani speaking homes: $\mathrm{OR}=3.2$, $95 \%$ CI 1.1-8.8), those who worked for money ( $\mathrm{OR}=4.3,95 \%$ CI 2.-9.5) and those who reported difficulties with studies $(\mathrm{OR}=3.0,95 \% \mathrm{CI}$ 1.4-6.5) had higher prevalence of mental disorders (see online table DS1).

\section{Neighbourhood}

Multivariate analysis found that distrust of the neighbourhood $(\mathrm{OR}=3.2,95 \% \mathrm{CI} 1.6-6.2)$, an unsafe neighbourhood $(\mathrm{OR}=4.8$, 95\% CI 2.4-9.6) and unavailability of health facilities within easy reach of home $(\mathrm{OR}=2.3,95 \% \mathrm{CI} 1.1-5.1)$ were independently associated with a mental disorder (see online table DS1).

\section{Peer relationships and leisure}

Most adolescents (78.8\%) reported having enough friends and frequently spending time with them (62.5\%). A small number (3.3\%) reported ever having had a boyfriend or girlfriend. Multivariate analysis found that ever having had a girlfriend or boyfriend to whom the adolescent was sexually attracted $(\mathrm{OR}=6.09$, 95\% CI 2.1-12.5) and a 'frequent' leisure score (as compared with a 'rare' score; $\mathrm{OR}=3.68,95 \%$ CI $1.5-9.3$ ) were independently associated with a mental disorder (see online table DS2).

\section{Parental relation and family support}

Multivariate analysis found that being able to talk easily with one's mother $(\mathrm{OR}=0.36,95 \%$ CI $0.1-0.97)$, reporting one's family as being the primary source of support (compared with rarely: $\mathrm{OR}=0.20,95 \% \mathrm{CI} 0.1-0.6)$, gender-based discrimination of girls $(\mathrm{OR}=3.71,95 \% \mathrm{CI} 1.2-11.3)$ and a medium or high parental stress score $(\mathrm{OR}=2.23,95 \% \mathrm{CI} 1.0-4.7$ and $\mathrm{OR}=2.54$, 95\% CI $0.7-8.6$ respectively) compared with a low score were independently associated with current mental disorder (see online table DS3).

\section{Substance use and violence}

A total of 37 participants (1.8\%) had used alcohol, 15 (0.7\%) had smoked tobacco and $30(1.5 \%)$ had chewed tobacco during the 
past 12 months. Frequent physical or verbal abuse from parents or other family members was reported from 315 participants (15.4\%). In addition, $283(13.8 \%)$ reported frequent abuse from teachers and 85 (4.2\%) from peers. At least one incident of being sexually abused was reported by 60 ( 20 males, 40 females; $2.9 \%$ ) participants. Multivariate analysis found that chewing tobacco $(\mathrm{OR}=5.7,95 \%$ CI 1.2-28.0) and physical/verbal abuse from parents or other family members $(\mathrm{OR}=3.50,95 \%$ CI $1.5-8.0)$ and peers $(\mathrm{OR}=2.9,95 \%$ CI $1.1-7.9)$ were independently associated with a mental disorder (see online table DS4).

\section{Multivariate model}

All factors independently associated with a mental disorder from domain-specific analyses were retained for further multivariate analyses. The final multivariate model identified a subset of factors which remained independently associated with a mental disorder after adjustment for all other factors (Table 1). Significantly higher prevalence of mental disorders were found among adolescents from English-speaking homes, unsafe neighbourhoods, reporting difficulties with studies, having had a girlfriend or boyfriend, having a 'frequent' leisure score and having frequently faced physical or verbal abuse from parents or other family members. Having one's family as the primary source of social support was associated with a lower prevalence of mental disorders.

\section{Discussion}

Prevalence of any current DSM-IV psychiatric disorder in this study was low $(1.81 \%$, 95\% CI $1.27 \%-2.48 \%)$. Emotional disorders were most common, followed by behavioural disorders and ADHD. The higher prevalence among those from an urban area and those with a middle or upper class lifestyle was reflected by the increased prevalence in English-speaking adolescents who adopt non-traditional leisure interests and have a close friend of the opposite gender. Apart from the expected association with recognised risk factors (sexual violence, parental physical abuse, difficulties with studies, tobacco) and protective factors (strong family support) for a mental disorder, we also found an association between gender discrimination, rooted in South Asian cultural values, in girls.

\section{Reasons for low prevalence of mental disorders}

A review of 52 studies in 20 countries conducted over 40 years reported a very wide range in prevalence of mental disorders in adolescents from $1 \%$ to $51 \%$. $^{2}$ Published studies from India similarly show a wide range of prevalence of psychiatric morbidity in adolescents and children ranging from $2.6 \%$ to $35.6 \% .{ }^{13-19}$ In our study, prevalence is lower than these and we propose three possible explanations for this: methodological factors, the scope of our study and the prevalence of protective factors. The instrument we used to assess mental disorders (DAWBA) is a structured interview which has been tested across many cultures and languages (including in South Asia). The DAWBA combines the strengths of a highly structured interview administered by trained lay interviewers with an experienced clinician's review to increase the clinical validity of the diagnosis. ${ }^{5}$ In a Bangladeshi sample, ${ }^{43}$ the observed association with independently assigned clinical diagnoses was as strong as in the UK, demonstrating similar predictive power and comparable validity of the algorithm across different cultures, languages and social backgrounds. ${ }^{44}$ Another strength of the DAWBA is that diagnosis depends on impact criteria (on various aspects of daily life) as well as symptoms. ${ }^{31}$ Prevalence is highly dependent on the extent to which the algorithms are used to make the diagnosis including functional impairment: ${ }^{45}$

\begin{tabular}{|c|c|c|c|}
\hline Risk factor & Odds ratio & $95 \% \mathrm{Cl}$ & $P$ \\
\hline Area & & & 0.90 \\
\hline Urban & 1.00 & & \\
\hline Rural & 0.94 & $0.3-2.6$ & \\
\hline Gender & & & 0.86 \\
\hline Male & 1.00 & & \\
\hline Female & 1.07 & $0.5-2.3$ & \\
\hline Age, years & & & 0.52 \\
\hline 12 & 1.00 & & \\
\hline 13 & 1.45 & $0.5-4.6$ & \\
\hline 14 & 2.36 & $0.8-6.9$ & \\
\hline 15 & 1.52 & $0.4-5.2$ & \\
\hline 16 & 1.12 & $0.3-4.1$ & \\
\hline Language spoken & & & 0.13 \\
\hline Konkani & 1.00 & & \\
\hline English & 3.45 & $1.1-10.3$ & \\
\hline Hindi & 1.59 & $0.5-4.9$ & \\
\hline Marathi and others & 0.80 & $0.2-3.1$ & \\
\hline Difficulty with studies & & & 0.03 \\
\hline No & 1.00 & & \\
\hline Yes & 2.52 & $1.1-5.7$ & \\
\hline Neighbourhood safety & & & $<0.001$ \\
\hline Safe & 1.00 & & \\
\hline Unsafe & 5.19 & $2.4-11.1$ & \\
\hline Ever had a boyfriend/girlffriend & & & 0.03 \\
\hline No & 1 & & \\
\hline Yes & 3.01 & $1.1-8.1$ & \\
\hline Leisure score ('non-traditional' lifestyle) & & & 0.02 \\
\hline Rare & 1.00 & & \\
\hline Moderate & 1.28 & $0.5-3.6$ & \\
\hline Frequent & 3.56 & $1.4-9.1$ & \\
\hline Chew tobacco & & & 0.05 \\
\hline No & 1.00 & & \\
\hline Yes & 5.51 & $1.0-30.7$ & \\
\hline Physical/verbal abuse from parents & & & 0.02 \\
\hline Never/rarely & 1.00 & & \\
\hline Occasionally & 1.5 & $0.6-3.7$ & \\
\hline Often & 2.90 & $1.3-6.8$ & \\
\hline Family support & & & 0.01 \\
\hline Rarely & 1.00 & & \\
\hline sometimes & 0.18 & $0.1-0.7$ & \\
\hline Often & 0.15 & $0.04-0.5$ & \\
\hline Always & 0.16 & $0.1-0.5$ & \\
\hline
\end{tabular}

defining disorder solely in terms of symptoms can result in implausibly high rates. ${ }^{3}$ For example, a study in south India showed a reduction in prevalence of mental disorders from $9.4 \%$ to $5.2 \%$ with the inclusion of an impairment criterion. ${ }^{14}$ In DSM-IV ${ }^{46}$ the most common child psychiatric disorders are now defined in terms of impact as well as symptoms: operational criteria stipulate that symptoms must result either in substantial distress for the child or in significant impairment in the child's ability to fulfil normal role expectations in everyday life. Our survey is one of the few from a low- or middle-income country to use the DSM-IV which emphasises significant distress or impairment. Finally, the translation and piloting of the DAWBA was carried out with great care - our interviewers were trained by an experienced user of the DAWBA in a low- or middle-income country setting and were also closely monitored during the fieldwork.

The DAWBA uses a one-stage design which requires an interview with the parent and child and, if possible, the administration of a teacher questionnaire. ${ }^{42}$ For generating the DAWBA diagnostic rating, we used 2048 adolescent interviews and parts of 1198 
parent DAWBA interviews. The lack of an interview with a substantial minority clearly affected the prevalence figure as we found that the prevalence of a disorder was almost twice as high in the group with parent and adolescent data compared with the group with adolescent data only. Hence partial absence of parent data and the total absence of teacher data could have also contributed to lower estimates of prevalence. Nevertheless, prevalence among those with both parent and adolescent data $(2.3 \%)$ is still low by global and Indian standards. Adolescents with a parental interview were more likely $(P<0.05)$ to be from rural areas, to be following non-Hindu religions, from Hindi-speaking homes, engaging in activities with parents and have two or more close friends. They were also more likely to live with their parents and not have a boyfriend or girlfriend. Some of these factors were associated with a mental disorder in our primary analysis; however, there is no reason to believe that the strengths of association of factors reported within the complete sample differ from those in the subsample with parental data.

The second reason for the low prevalence may be the scope of our study, notably our selection of a narrow age band and the type of mental disorders assessed. In previous studies, prevalence estimates of mental disorders have included uncomplicated enuresis, learning disabilities and neurological conditions like epilepsy. The primary focus of our study was emotional disorders, behavioural disorders and ADHD based on DSM-IV criteria. ${ }^{46}$ The most comparable study is a recent survey from south India ${ }^{13}$ which also reported a relatively low prevalence of emotional and conduct disorders of $3.9 \%$ among 4 - to 16-year-olds. Another study from south India ${ }^{47}$ reported a $3 \%$ prevalence of depression among school-attending adolescents (13-19 years). Higher rates are generally reported in older adolescents ( $>16$ years), who are experiencing a more rapid transition to adulthood, accompanied by several potentially stressful life events (including marriage). Research by our group in India has reported a prevalence of depressive and anxiety disorders of $6-7 \%$ in women aged 18 years and above ${ }^{30}$ and found that married women comprise a high-risk group compared with single women. It is possible that the role transition from childhood, which in this cultural milieu may extend well into adolescence (exemplified, for example, by high levels of parental authority in decision making) to adulthood, often heralded by marriage, is frequently sudden and without the gradual development of autonomy seen in some other cultures. While the persistence of a 'child' role into adolescence may be a protective factor in early adolescence, the sudden role transition may be a key factor in explaining the increased vulnerabilities for young, married adults.

Although studies carried out in other low- and middle-income countries using the DAWBA have reported higher prevalence, for example, $15 \%$ among 5 - to 10 -year olds in Bangladesh ${ }^{43}$ and $12.8 \%$ among 11 - to 14 -year olds in south-east Brazil, ${ }^{39}$ our findings of low prevalence are similar to those of a UK national survey in which children of Indian families had a relatively low prevalence of mental disorders (3\% compared with $7-10 \%$ in other ethnic groups). ${ }^{42}$ Thus, our third possible explanation for the low prevalence is that this is a true finding reflecting the role of protective factors, in particular the relationship of the adolescent with his or her family. The role of the family in promoting mental health of adolescents is clearly demonstrated by the independent protective effect of family support. Our study found an association of mental disorders with urban, middle class and non-traditional lifestyles which have been adopted by some adolescents; thus, we found an increased prevalence among English-speaking adolescents, having non-traditional interests (such as going to the disco) and having an intimate friend of the opposite gender. We hypothesise that these lifestyles lead to an increased risk of conflict with traditional values, creating stressful environments that predispose adolescents to mental disorders. This hypothesis would support suggestions that children's mental health may be adversely affected by a non-traditional value system that promotes individualism, weakens social ties and creates ambivalence towards children. ${ }^{9}$ On the other hand, we note that certain traditional practices rooted in Indian culture which facilitate gender discrimination (against girls) could be detrimental to mental health of girls. Furthermore, parental physical violence, sexual abuse, difficulties with studies and tobacco use are risk factors that are universal. Other studies have also indicated that 'parent hostility, inconsistent and harsh coercive discipline have been consistently linked to externalising behaviour. ${ }^{48}$

\section{Limitations}

We acknowledge a number of limitations in our research which may have biased our findings. A lower rate of disorder may indicate that the DSM-IV may not be fully capturing mental distress in this cultural context and we propose that ethnographic investigations into cultural expressions of child mental distress are warranted. The composite scores we generated to explain the factors associated with mental disorder, though derived from the raw data and having face validity, were not a priori constructs. Thus, our proposed ethnographic investigations should also consider the appropriateness of integration of the individual factors in each composite variable. In addition, we propose the need for hypothesis-driven research, testing the association between these constructs and the risk of mental disorders. The likelihood of reverse causality cannot be excluded as a result of our cross-sectional study design. The higher non-participation rate from urban areas may have introduced a selection bias, but re-running models with sampling weights to allow for the differential response rates did not change our main findings. The possibility of a response bias due to differential response to the DAWBA by adolescents from traditional and non-traditional backgrounds, which in turn may have led to a spurious increase in rates reported in the latter group, cannot be ruled out. The partial absence of parent data and the total absence of teacher data may have led us to underestimate the prevalence. Factors other than family support, which we have not measured, including biologically determined risk and resilience factors, may have contributed to low prevalence of mental disorders. Goa is a relatively rich state with better socio-economic indicators than most other parts of India and our study may not be generalisable to other regions of the country. On the other hand, ours is the largest population-based survey of adolescent mental health in India that utilised a structured diagnostic interview and assessed a range of contextual risk factors.

\section{Future research}

In the context of rapid social change being witnessed in India, we suggest that there is a need to research the appropriate parenting models which may play a role in preventing mental disorders in early adolescence while also building resilience into adulthood.

\footnotetext{
Aravind Pillai, MBBS, MPH, Sangath, Goa, India, Vikram Patel, PhD, London Schoo of Hygiene and Tropical Medicine, UK, and Sangath, Goa, Percy Cardozo, MA, Sangath, Goa, India, Robert Goodman, PhD, FRCPsych, MRCP, Kings College London Institute of Psychiatry, UK, Helen A. Weiss, PhD, London School of Hygiene and Tropical Medicine, UK, Gracy Andrew, MA, Sangath, Goa, India.

Correspondence: Vikram Patel, PhD, London School of Hygiene and Tropica Medicine, UK. Email: vikram.patel@|shtm.ac.uk

First received 3 December 2006, final version 3 October 2007, accepted 17 October 2007
} 


\section{Acknowledgements}

We acknowledge the role of the WHO's Special Programme of Research, Development and Research Training in Human Reproduction in funding the study. V.P. is supported by Wellcome Trust Senior Clinical Research Fellowship in Tropical Medicine. We than Jayashree Ramakrishna MPH Additional Professor and Head, Department of Health their valuable inputs during the first phase of the study. We are grateful to all the schoo principals in the study areas, the Department of Education, Government of Goa, who gave us permission to carry out the study in schools, the panchayat and other community members in the rural areas, and the staff of the Bali primary health centre for playing a key role in helping us receive community sanctions. Finally, we acknowledge the contribution of the research team of the Mitr project, the administrative support from Sangath and all the young people and their parents who participated in this study.

\section{References}

1 Elster AB, Marcell AV. Health care of adolescent males: overview, rationale and recommendations. Adolesc Med 2003; 14: 525-40.

2 Roberts RE, Attkisson CC, Rosenblatt A. Prevalence of psychopathology among children and adolescents. Am J Psychiatry 1998; 155: 715-25.

3 Melzer H, Gatward R, Goodman R, Ford T. The Mental Health of Children and Adolescents in Great Britain. TSO (The Stationery Office), 2000.

4 Shaffer D, Fisher $P$, Dulcan M, Dulcan MK, Davies M, Piacentini J, SchwabStone ME, Lakey BB, Bourdon K, Jensen PS, Bird HR, Canino G, Regier DA. The NIMH Diagnostic Interview Schedule for Children Version 2.3 (DISC 2.3): Description; acceptability; prevalence rates; and performance in the MECA study. J Am Acad Child Adolesc Psychiatry 1996; 35: 865-77.

5 Ford T, Goodman R, Meltzer H. The British Child and Adolescent Mental Health Survey 1999: the prevalence of DSM-IV disorders. J Am Acad Child Adolesc Psychiatry 2003; 42: 1203-11.

6 Boys A, Farrell M, Taylor C, Marsden J, Goodman R, Brugha T, Bebbington P Jenkins $\mathrm{R}$, Meltzer $\mathrm{H}$. Psychiatric morbidity and substance use in young people aged 13-15 years: results from the Child and Adolescent Survey of Mental Health. Br J Psychiatry 2003; 182: 509-17.

7 Patel V, Flisher A, Hetrick S, McGorry P. Mental health of young people: a global public-health challenge. Lancet 2007; 369: 1302-13.

8 World Health Organization. Programming for Adolescent Health and Development: Technical Report, number 886. WHO, 1999.

9 Timimi S. Effect of globalisation on children's mental health. BMJ 2005; 331 37-9.

10 Remschmidt $\mathrm{H}$, Belfer $\mathrm{M}$. Mental health care for children and adolescents worldwide: a review. World Psychiatry 2005; 4: 147-53.

11 Registrar General of India. Population projection for India and States, 1996-2016. Report of the Technical Group of Population Projection. Planning Commission, New Delhi, 1996.

12 Aaron R, Joseph A, Abraham S, Muliyil J, George K, Prasad J, Minz S, Abraham VJ, Bose A. Suicides in young people in rural southern India. Lancet 2004; 363: 1117-18.

13 Srinath S, Girimaji SC, Gururaj G, Seshadri S, Subbakrishna DK, Bhola P. Kumar N. Epidemiological study of child and adolescent psychiatric disorders in urban and rural areas of Bangalore, India. Indian J Med Res 2005; 122: 67-79.

14 Hackett R, Hackett L, Bhakta P, Gowers S. The prevalence and associations of psychiatric disorder in children in Kerala, South India. J Child Psychol Psychiatry 1999; 40: 801-7.

15 Sethi B, Gupta S, Kumar R. 300 urban families: a psychiatric survey. Indian J Psychiatry 1967; 9: 280-302.

16 Sethi B, Gupta C, Kumar R, Kumar P. A psychiatric survey of 500 rural families. Indian J Psychiatry 1972; 14: 183-96.

17 Lal N, Sethi B. Estimate of mental ill health in children in an urban community. Indian J Pediatr 1977; 4: 55-64.

18 Nandi D, Ajmany S, Ganguly H, Banerjeee G, Boral G, Ghosh A. Psychiatric disorder in a rural community in West Bengal - an epidemiology study. Indian J Psychiatry 1975; 17: 87-9.

19 Verghese A, Beig A. Psychiatric disturbances in children: an epidemiological study. Indian J Med Res 1974; 62: 1538-42.

20 Hoare P, Will D, Wrate R. Forfar and Arneil's Textbook of Pediatrics (5th edn). Churchill Livingstone, 1998.

21 Beam MR, Gil-Rivas V, Greenberger E, Chen C. Adolescent problem behavio and depressed mood risk and protection within and across social contexts. $J$ Youth Adolescence 2002; 31: 343-57.

22 Gureje 0 , Omigbodun 00 . Children with mental disorders in primary care: functional status and risk factors. Acta Psychiatr Scand 1995; 92: 310-14.
23 Rahim SI, Cederblad M. Epidemiology of mental disorders in young adults of a newly urbanised area in Khartoum, Sudan. Br J Psychiatry 1989; 155: 44-7.

24 Chandra R, Srinivasan S, Chandrasekaran R, Mahadevan S. The prevalence of mental disorders in school-age children attending a general paediatric department in southern India. Acta Psychiatr Scand 1993; 87: 192-6.

25 Young SE, Mikulich SK, Goodwin MB, Hardy J, Martin CL, Zoccolillo MS, Crowley TJ. Treated delinquent boys' substance use: onset, pattern, relationship to conduct and mood disorders. Drug Alcohol Depend 1995; 37: 149-62.

26 Bagley C, Mallick K. Prediction of sexual, emotional, and physical maltreatment and mental health outcomes in a longitudinal cohort of 290 adolescent women. Child Maltreat 2000; 5: 218-26.

27 Krupnick JL, Green BL, Stockton P, Goodman L, Corcoran C, Petty R. Mental health effects of adolescent trauma exposure in a female college sample: exploring differential outcomes based on experiences of unique trauma types and dimensions. Psychiatry 2004; 67: 264-79.

28 Registrar General \& Census Commissioner, India. Census of India 2001. http://www.censusindia.gov.in

29 Andrew G, Patel V, Ramakrishna J. Sex, studies or strife? What to integrate in adolescent health services. Reprod Health Matters 2003; 11: 120-9.

30 Patel V, Kirkwood BR, Pednekar S, Pereira B, Barros P, Fernandes J, Datta J, Pai R, weiss $H$, Mabey D. Gender disadvantage and reproductive health risk factors for common mental disorders in women: a community survey in India. Arch Gen Psychiatry 2006; 63: 404-13.

31 Hackett R, Hackett L. Child psychiatry across cultures. Int Rev Psychiatry 1999; 11: 225-35.

32 Rahi M, Kumavat AP, Garg S, Singh MM. Socio-demographic correlates of psychiatric disorders. Indian J Pediatr 2005; 72: 395-8.

33 Blum RW, Beuhring T, Shew ML, Bearinger LH, Sieving RE, Resnick MD. The effects of race/ethnicity, income, and family structure on adolescent risk behaviors. Am J Public Health 2000; 90: 1879-84.

34 Bhui K, Stansfeld S, Head J, Haines M, Hillier S, Taylor S, Viner R, Booy R. Cultural identity, acculturation, and mental health among adolescents in east London's multiethnic community. J Epidemiol Community Health 2005; 59: 296-302.

35 Khurana S, Sharma N, Jena S, Saha R, Ingle G. Mental health status of runaway adolescents. Indian J Pediatr 2004; 71: 405-9.

36 Goodman R, Ford T, Richards H, Gatward R, Meltzer H. The Development and Well-Being Assessment: description and initial validation of an integrated assessment of child and adolescent psychopathology. J Child Psychol Psychiatry 2000; 41: 645-55.

37 World Health Organization. The ICD-10 Classification of Mental and Behavioural Disorders. WHO, 1992.

38 American Psychiatric Association. Diagnostic Criteria from DSM-IV (Indian edition). Jaypee Brothers Medical Publishers, 1994.

39 Fleitlich-Bilyk B, Goodman R. Prevalence of child and adolescent psychiatric disorders in southeast Brazil. J Am Acad Child Adolesc Psychiatry 2004; 43 727-34.

40 Goodman R, Neves dos Santos D, Robatto Nunes AP, Pereira de Miranda D, Fleitlich-Bilyk B, Almeida Filho N. The Ilha de Mare study: a survey of child mental health problems in a predominantly African-Brazilian rural community. Soc Psychiatry Psychiatr Epidemiol 2005; 40: 11-17.

41 Goodman R, Slobodskaya H, Knyazev G. Russian child mental health - a cross-sectional study of prevalence and risk factors. Eur Child Adolesc Psychiatry 2005; 14: 28-33.

42 Department of Health. Mental Health of Children and Young People in Great Britain, 2004. Palgrave MacMillan, 2005.

43 Mullick MS, Goodman R. The prevalence of psychiatric disorders among 5-10 year olds in rural, urban and slum areas in Bangladesh: an exploratory study. Soc Psychiatry Psychiatr Epidemiol 2005; 40: 663-71.

44 Woerner W, Fleitlich-Bilyk B, Martinussen R, Fletcher J, Cucchiaro G, Dalgalarrondo $P$, Lui $M$, Tamrock R. The Strengths and Difficulties Questionnaire overseas: evaluations and applications of the SDQ beyond Europe. Eur Child Adolesc Psychiatry 2004; 13 Suppl 2: II47-54.

45 Costello EJ, Egger H, Angold A. 10-year research update review: the epidemiology of child and adolescent psychiatric disorders: II. Methods and public health burden. J Am Acad Child Adolesc Psychiatry 2005; 44: 972-86.

46 American Psychiatric Association. DSM-IV: Diagnostic and Statistical Manual of Mental Disorders (4th edition). American Psychiatric Association, 1994.

47 Nair MK, Paul MK, John R. Prevalence of depression among adolescents. Indian J Pediatr 2004; 71: 523-4.

48 Stern SB, Smith CA, Jang SJ. Urban families and adolescent mental health. Soc Work Res 1999; 23: 15-27.

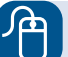

